\title{
Riesgo de sesgo: ¿calidad o validez?
}

\author{
Dr. Enrique Ardila \\ Editor jefe \\ Revista Colombiana de Endocrinología, \\ Diabetes y Metabolismo
}

Apreciado doctor Ardila:

Los ensayos clínicos aleatorizados (ECA) denotan el grado más alto de investigación científica por su gran nivel de evidencia, siendo sus resultados importantes a la hora de instaurar una nueva terapia farmacológica o intervención clínica en pacientes (1); no obstante, el aumento en los últimos años de este tipo de investigaciones científicas nos obliga a ser rigurosos y un tanto minucioso a la hora de seleccionar el mejor estudio de investigación, por lo que se debe diferenciar entre "evaluación de la calidad" y "riesgo de sesgo".

Dichos conceptos deben ser explícitamente descritos y visualmente diferenciados en la metodología de cualquier revisión sistemática, es por ello que tomando como referencia lo publicado por Chacón K., donde menciona que la "calidad de los estudios fue evaluada con la herramienta de evaluación de riesgo de sesgo de Cochrane" (2), se determina que la terminología utilizada no es pertinente, puesto que la "calidad" hace referencia a la elaboración detallada y técnica o hasta qué punto se ha realizado un estudio bajo los mejores estándares posibles, pero no quiere decir que se haya evitado el riesgo de sesgo en los resultados o qué tanta credibilidad tengan estos, por lo tanto, el riesgo de sesgo abarcaría esta incógnita (3), siendo una herramienta que sirve para evaluar la validez de los ECA (4), recomendamos en próximos estudios sustituir la palabra "calidad" por "validez" para evitar confusiones metodológicas.

\section{José Rafael Villarreal Escorcia(D)} Universidad del Sinú, Cartagena, Colombia Correo electrónico: linkin3187@gmail.com

\section{Referencias}

[1] Bachelet VC, Pardo-Hernández $\mathrm{H}$. Quality of reporting and risk of bias of randomized clinical trials published in Spanish and Latin American journals. Medwave. 2019;19(1):e7573. https://doi.org/10.5867/ medwave.2019.01.7573

[2] Chacón K, Mendivelso F, Gómez O, González C, Pinto D, Yama E, et al. Efectividad y seguridad comparada de inhibidores del cotransportador sodio-glucosa tipo 2 (iSGLT2) en diabetes mellitus tipo 2: revisión rápida de revisiones sistemáticas 
y metaanálisis. Rev Col End. 2020 dic.;7(4):222-34. https://doi. org/ 10.53853/encr.7.4.648

[3] Urrútia G, Bonfill X. Declaración PRISMA: una propuesta para mejorar la ubicación de revisiones sistemáticas y metaanálisis. Med Clin. 2010;135(1):507-11. https://doi. org/10.1016/j.medcli.2010.01.015
[4] Palacios MA, Ojeda-Gómez RC, Ticse-Huaricancha IL, CajachaguaHilario K. Critical analysis of randomized clinical trials: The risk of bias. Rev Estomatol Herediana. 2015 oct-dic;25(4):304-8. https:// doi.org/ 10.20453/reh.v25i4.2740 